\title{
A New Extension of Serrin's Lower Semicontinuity Theorem
}

\author{
Xiaohong Hu${ }^{1,2}$ and Shiqing Zhang ${ }^{2}$ \\ ${ }^{1}$ School of Mathematics and Physics, Chongqing University of Posts and Telecommunications, Chongqing 400065, China \\ ${ }^{2}$ Mathematical College, Sichuan University, Chengdu 610064, China
}

Correspondence should be addressed to Xiaohong Hu; huxh@cqupt.edu.cn

Received 18 February 2013; Revised 3 June 2013; Accepted 3 June 2013

Academic Editor: Yisheng Song

Copyright (C) $2013 \mathrm{X}$. Hu and S. Zhang. This is an open access article distributed under the Creative Commons Attribution License, which permits unrestricted use, distribution, and reproduction in any medium, provided the original work is properly cited.

We present a new extension of Serrin's lower semicontinuity theorem. We prove that the variational functional $\int_{\Omega} f\left(x, u, u^{\prime}\right) d x$ defined on $W_{\text {loc }}^{1,1}(\Omega)$ is lower semicontinuous with respect to the strong convergence in $L_{\text {loc }}^{1}$, under the assumptions that the integrand $f(x, s, \xi)$ has the locally absolute continuity about the variable $x$.

\section{Introduction and Main Results}

The aim of this paper is to give some new sufficient conditions for lower semicontinuity with respect to the strong convergence in $L_{\text {loc }}^{1}$ for integral functionals

$$
F(u, \Omega)=\int_{\Omega} f(x, u(x), D u(x)) d x,
$$

where $\Omega$ is an open set of $R^{n}, u \in W_{\text {loc }}^{1,1}(\Omega)$, defined on $W_{\mathrm{loc}}^{1,1}(\Omega)=\left\{u: u \in L^{1}(K), D u \in L^{1}(K)\right.$, for all $\left.K \subset \subset \Omega\right\}$ [1], $D u$ denotes the generalized gradient of $u$, and the integrand $f(x, s, \xi): \Omega \times R \times R^{n} \rightarrow[0, \infty)$ satisfies the following condition:

(H1) $f$ is continuous in $\Omega \times R \times R^{n}$, and $f(x, s, \xi)$ is convex in $\xi \in R^{n}$ for any fixed $(x, s) \in \Omega \times R$.

The integral functional $F$ is called lower semicontinuous in $W_{\text {loc }}^{1,1}(\Omega)$ with respect to the strong convergence in $L_{\text {loc }}^{1}$, if, for every $u_{m}, u \in W_{\text {loc }}^{1,1}(\Omega)$, such that $u_{m} \rightarrow u$ in $L_{\text {loc }}^{1}$, then

$$
\liminf _{m \rightarrow+\infty} F\left(u_{m}, \Omega\right) \geq F(u, \Omega) .
$$

It is well known that condition (H1) is not sufficient for lower semicontinuity of the integral $F$ in (1) (see book [2]). In addition to (H1), Serrin [3] proposed some sufficient conditions for lower semicontinuity of the integral $F$. One of the most known conclusions is the following one.
Theorem 1 (see [3]). In addition to (H1), iff satisfies one of the following conditions:

(a) $f(x, s, \xi) \rightarrow+\infty$ when $|\xi| \rightarrow+\infty$, for all $(x, s) \in$ $\Omega \times R$,

(b) $f(x, s, \xi)$ is strictly convex in $\xi \in R^{n}$ for all $(x, s) \in$ $\Omega \times R$,

(c) the derivatives $f_{x}(x, s, \xi), f_{\xi}(x, s, \xi)$, and $f_{\xi x}(x, s, \xi)$ exist and are continuous for all $(x, s, \xi) \in \Omega \times R \times R^{n}$.

then $F(u, \Omega)$ is lower semicontinuous in $W_{\text {loc }}^{1,1}(\Omega)$ with respect to the strong convergence in $L_{\mathrm{loc}}^{1}$.

Conditions (a), (b), and (c) quoted above are clearly independent, in the sense that we can find a continuous function $f$ satisfying just one of them but none of the others. Many scholars have weakened the conditions of integrand $f$ and generalized Theorem 1, such as Ambrosio et al. [4], Cicco and Leoni [5], Fonseca and Leoni [6, 7]. In particular Gori et al. $[8,9]$ proved the following theorems.

Theorem 2 (see $[8,9])$. Let one assume that $f$ satisfies (H1) and that, for every compact set $K \subset \Omega \times R \times R^{n}$, there exists a constant $L=L(K)$ such that

$$
\begin{array}{r}
\left|f_{\xi}\left(x_{1}, s, \xi\right)-f_{\xi}\left(x_{2}, s, \xi\right)\right| \leq L\left|x_{1}-x_{2}\right|, \\
\forall\left(x_{1}, s, \xi\right),\left(x_{2}, s, \xi\right) \in K,
\end{array}
$$


and, for every compact set $K_{1} \subset \Omega \times R$, there exists a constant $L_{1}=L_{1}\left(K_{1}\right)$ such that

$$
\begin{array}{r}
\left|f_{\xi}(x, s, \xi)\right| \leq L_{1}, \quad \forall(x, s) \in K_{1}, \quad \forall \xi \in R^{n}, \\
\left|f_{\xi}\left(x, s, \xi_{1}\right)-f_{\xi}\left(x, s, \xi_{2}\right)\right| \leq L_{1}\left|\xi_{1}-\xi_{2}\right|, \\
\forall(x, s) \in K_{1}, \quad \forall \xi_{1}, \xi_{2} \in R^{n} .
\end{array}
$$

Then $F(u, \Omega)$ is lower semicontinuous in $W_{\text {loc }}^{1,1}(\Omega)$ with respect to the strong convergence in $L_{\text {loc }}^{1}$.

Theorem 3 (see $[8,9]$ ). Let $f$ satisfy (H1) such that, for every open set $\Omega^{\prime} \times H \times K \subset \subset \Omega \times R \times R^{n}$, there exists a constant $L=L_{\Omega^{\prime} \times H \times K}$ such that, for every $x_{1}, x_{2} \in \Omega^{\prime}, s \in H$, and $\xi \in K$,

$$
\left|f\left(x_{1}, s, \xi\right)-f\left(x_{2}, s, \xi\right)\right| \leq L\left|x_{1}-x_{2}\right| .
$$

Then the functional $F(u, \Omega)$ is lower semicontinuous in $W_{\text {loc }}^{1,1}(\Omega)$ with respect to the $L_{\text {loc }}^{1}$ convergence.

Condition (5) means that $f$ is locally Lipschitz continuous with respect to $x$, that is, the Lipschitz constant is not uniform for $(s, \xi) \in R \times R^{n}$. This is an improvement of (c) of Serrin's Theorem 1. Then a question arises, that is whether there are weaker enough conditions more than locally Lipschitz continuity. In this paper, we consider absolute continuity. Obviously, absolute continuity is weaker than Lipschitz continuity. The following theorems show that, in addition to (H1), the locally absolute continuity on $f$ about $x$ is sufficient for the lower semicontinuity of the variational functional.

Theorem 4. Let $\Omega \subset R$ be an open set; $f(x, s, \xi): \Omega \times R \times R \rightarrow$ $[0,+\infty)$ satisfies the following conditions:

(H1) $f(x, s, \xi)$ is continuous on $\Omega \times R \times R$, and, $f(x, s, \xi)$ is convex in $\xi \in R$ for all $(x, s) \in \Omega \times R$;

(H2) $f_{\xi}(x, s, \xi)$ is continuous on $\Omega \times R \times R$, and for every compact set of $\Omega \times R \times R, f_{\xi}(x, s, \xi)$ is absolutely continuous about $x$;

(H3) for every compact set $K_{1} \subset \Omega \times R$, there exists a constant $L_{1}=L_{1}\left(K_{1}\right)$, such that

$$
\begin{array}{r}
\left|f_{\xi}\right| \leq L_{1}, \quad \forall(x, s) \in K_{1}, \quad \forall \xi \in R, \\
\left|f_{\xi}\left(x, s, \xi_{1}\right)-f_{\xi}\left(x, s, \xi_{2}\right)\right| \leq L_{1}\left|\xi_{1}-\xi_{2}\right|, \\
\forall(x, s) \in K_{1}, \quad \forall \xi_{1}, \xi_{2} \in R .
\end{array}
$$

Then the functional $F(u, \Omega)=\int_{\Omega} f\left(x, u(x), u^{\prime}(x)\right) d x$ is lower semicontinuous in $W_{\text {loc }}^{1,1}(\Omega)$ with respect to the strong convergence in $L_{l o c}^{1}(\Omega)$.

Theorem 5. Let $\Omega \subset R$ be an open set; $f(x, s, \xi): \Omega \times R \times R \rightarrow$ $[0,+\infty)$ satisfies $(H 1)$ and the following condition:

(H4) for every compact set of $\Omega \times R \times R, f(x, s, \xi)$ is absolutely continuous about $x$.
Then the functional $F(u, \Omega)$ is lower semicontinuous in $W_{\text {loc }}^{1,1}(\Omega)$ with respect to the strong convergence in $L_{\text {loc }}^{1}(\Omega)$.

\section{Preliminaries}

In this section, we will collect some basic facts which will be used in the proofs of Theorems 4 and 5.

It is well know that a real function $f:[a, b] \rightarrow R$ is called an absolutely continuous function on $[a, b]$, if, for all $\varepsilon>$ $0, \exists \delta>0$, such that for any finite disjoint open interval $\left\{\left(a_{i}, b_{i}\right)\right\}_{i=1}^{n}$ on $[a, b]$, when $\sum_{i=1}^{n}\left(b_{i}-a_{i}\right)<\delta$, we have

$$
\sum_{i=1}^{n}\left|f\left(b_{i}\right)-f\left(a_{i}\right)\right|<\varepsilon .
$$

Obviously, if $f(x)$ is Lipschitz continuous on $[a, b], f(x)$ is absolutely continuous on $[a, b]$.

One of the main tool, used in the present paper, in order to prove the lower semicontinuity of the functional $F(u, \Omega)$ in (1), is an approximation result for convex functions due to De Giorgi [10].

Lemma 6 (see [10]). Let $U \subseteq R^{d}$ be an open set and $f: U \times$ $R^{n} \rightarrow[0,+\infty)$ a continuous function with compact support on $U$, such that, for every $t \in U, f(t, \cdot)$ is convex on $R^{n}$. Then there exists a sequence $\left\{\eta_{q}\right\}_{q=1}^{\infty} \subseteq C_{c}^{\infty}\left(R^{n}\right), \eta_{q} \geq 0, \int_{R^{n}} \eta_{q} d \rho=$ 1 , and $\operatorname{supp}\left(\eta_{q}\right) \subseteq B(0,1)$, such that, if we let

$$
\begin{gathered}
a_{q}(t)=\int_{R^{n}} f(t, \rho)\left\{(n+1) \eta_{q}(\rho)+\left\langle\nabla \eta_{q}(\rho), \rho\right\rangle\right\} d \rho, \\
b_{q}(t)=-\int_{R^{n}} f(t, \rho) \nabla \eta_{q}(\rho) d \rho,
\end{gathered}
$$

one has

$$
f_{j}(t, \xi)=\max _{1 \leq q \leq j}\left\{0, a_{q}(t)+\left\langle b_{q}(t), \xi\right\rangle\right\}, \quad j \in N,
$$

satisfying the following results:

(i) for every $j \in N, f_{j}: U \times R^{n} \rightarrow[0,+\infty)$ is a continuous function with compact support on $U$ such that, for all $t \in U, f_{j}(t, \cdot)$ is convex on $R^{n}$. Moreover, for all $(t, \xi) \in U \times R^{n}, f_{j}(t, \xi) \leq f_{j+1}(t, \xi)$, and

$$
f(t, \xi)=\sup _{j \in N} f_{j}(t, \xi)
$$

(ii) for every $j \in N$, there exists a constant $M_{j}>0$, such that, for all $(t, \xi) \in U \times R^{n}$,

$$
\left|f_{j}(t, \xi)\right| \leq M_{j}(1+|\xi|)
$$

and, for all $t \in U$, and $\xi_{1}, \xi_{2} \in R^{n}$;

$$
\left|f_{j}\left(t, \xi_{1}\right)-f_{j}\left(t, \xi_{2}\right)\right| \leq M_{j}\left|\xi_{1}-\xi_{2}\right| \text {. }
$$




\section{Proof of Theorem 4}

We will divide four steps to complete the proof of Theorem 4 .

Step 1. Let $\left\{\beta_{i}(x, s)\right\}_{i \in N}$ be a sequence of smooth functions satisfying

(1) there exists a compact set $\Omega^{\prime} \times H \subset \subset \Omega \times R$, such that $\beta_{i}(x, s)=0$, for all $(x, s) \in\left(\Omega \backslash \Omega^{\prime}\right) \times(R \backslash H)$;

(2) for every $i \in N, \beta_{i}(x, s) \leq \beta_{i+1}(x, s)$, for all $(x, s) \in$ $\Omega^{\prime} \times H$

(3) $\lim _{i \rightarrow+\infty} \beta_{i}(x, s)=1$, for all $(x, s) \in \Omega^{\prime} \times H$.

Let

$$
f_{i}(x, s, \xi)=\beta_{i}(x, s) f(x, s, \xi), \quad i=1,2, \ldots .
$$

It is clear that, for each $i \in N, f_{i}$ satisfies all the hypotheses in Theorem 4 and also vanishes if $(x, s)$ is outside $\Omega^{\prime} \times H$; thus

$$
\begin{gathered}
\lim _{i \rightarrow+\infty} f_{i}(x, s, \xi)=f(x, s, \xi), \quad \forall(x, s, \xi) \in \Omega^{\prime} \times H \times R, \\
f_{i}(x, s, \xi) \leq f_{i+1}(x, s, \xi) \leq f(x, s, \xi), \\
\forall i \in N, \quad \forall(x, s, \xi) \in \Omega^{\prime} \times H \times R .
\end{gathered}
$$

By Levi's Lemma, we have

$$
\lim _{i \rightarrow+\infty} \int_{\Omega^{\prime}} f_{i}(x, s, \xi) d x=\int_{\Omega^{\prime}} f(x, s, \xi) d x .
$$

Thus, without loss of generality, we can assume that there exists an open set $\Omega^{\prime} \times H \subset \subset \Omega \times R$, such that

$$
f(x, s, \xi)=0, \quad \forall(x, s, \xi) \in\left(\Omega \backslash \Omega^{\prime}\right) \times(R \backslash H) \times R .
$$

Let $u_{m}, u \in W_{\text {loc }}^{1,1}(\Omega)$ such that $u_{m} \rightarrow u$ in $L_{\text {loc }}^{1}(\Omega)$. We will prove that

$$
\liminf _{m \rightarrow+\infty} F\left(u_{m}, \Omega\right) \geq F(u, \Omega) .
$$

Without loss of generality, we can assume that

$$
\liminf _{m \rightarrow+\infty} F\left(u_{m}, \Omega\right)=\lim _{m \rightarrow+\infty} F\left(u_{m}, \Omega\right)<+\infty .
$$

By (17), we have $F\left(u_{m}, \Omega\right)=F\left(u_{m}, \Omega^{\prime}\right)$ and $F(u, \Omega)=$ $F\left(u, \Omega^{\prime}\right)$; thus we will only prove the following inequality:

$$
\lim _{m \rightarrow+\infty} F\left(u_{m}, \Omega^{\prime}\right) \geq F\left(u, \Omega^{\prime}\right) .
$$

Step 2. Let $\eta_{\varepsilon} \in C_{c}^{\infty}(R)$ be a mollifier, and, for $\epsilon>0$, define

$$
\begin{aligned}
v_{\varepsilon}(x) & =\eta_{\varepsilon} * u(x) \\
& =\int_{\Omega} \eta_{\varepsilon}(x-y) u(y) d y, \quad x \in\left[\Omega_{\varepsilon}\right],
\end{aligned}
$$

where $\left[\Omega_{\varepsilon}\right] \triangleq\{x \in \Omega: \operatorname{dist}(x, \partial \Omega)>\varepsilon\}$. We have

$$
\begin{aligned}
& {\left[u_{\varepsilon}(x)\right]^{\prime} }=\left[\int_{\Omega} \eta_{\varepsilon}(x-y) u(y) d y\right]_{x} \\
&=\int_{\Omega}\left[\eta_{\varepsilon}(x-y)\right]_{x} u(y) d y \\
&=\int_{B(x, \varepsilon)} \eta_{\varepsilon}(x-y)[u(y)]_{y} d y=\left[u^{\prime}\right]_{\varepsilon}(x), \\
& x \in \Omega_{\varepsilon} .
\end{aligned}
$$

In the following, we denote the derivative of $u_{\varepsilon}$ by $u_{\varepsilon}^{\prime}$. When $u \in W_{\text {loc }}^{1,1}(\Omega)$, we know $u^{\prime} \in L_{\text {loc }}^{1}(\Omega)$. By the properties of convolution, we know $u_{\varepsilon}^{\prime} \in C_{0}^{\infty}(\Omega)$ and

$$
u_{\varepsilon}^{\prime} \longrightarrow u^{\prime} \quad \text { in } L_{\mathrm{loc}}^{1}(\Omega) \text { as } \varepsilon \longrightarrow 0^{+},
$$

That is, for all $\delta>0, \exists \epsilon>0$, such that

$$
\int_{\Omega^{\prime}}\left|u_{\varepsilon}^{\prime}-u^{\prime}\right| d x<\delta
$$

Now we estimate the difference for the integrand values on different points:

$$
\begin{aligned}
f\left(x, u_{m}, u_{m}^{\prime}\right)-f\left(x, u, u^{\prime}\right) \\
=f\left(x, u_{m}, u_{m}^{\prime}\right)-f\left(x, u_{m}, u_{\varepsilon}^{\prime}\right) \\
+f\left(x, u_{m}, u_{\varepsilon}^{\prime}\right)-f\left(x, u, u_{\varepsilon}^{\prime}\right) \\
+f\left(x, u, u_{\varepsilon}^{\prime}\right)-f\left(x, u, u^{\prime}\right) .
\end{aligned}
$$

By the convexity of $f(x, s, \xi)$ with respect to $\xi$, we have

$$
\begin{aligned}
f\left(x, u_{m}, u_{m}^{\prime}\right)-f\left(x, u_{m}, u_{\varepsilon}^{\prime}\right) \\
\geq f_{\xi}\left(x, u_{m}, u_{\varepsilon}^{\prime}\right) \cdot\left(u_{m}^{\prime}-u_{\varepsilon}^{\prime}\right) . \\
=f_{\xi}\left(x, u_{m}, u_{\varepsilon}^{\prime}\right) \cdot u_{m}^{\prime}-f_{\xi}\left(x, u, u_{\varepsilon}^{\prime}\right) \cdot u^{\prime} \\
\quad+f_{\xi}\left(x, u, u_{\varepsilon}^{\prime}\right) \cdot\left(u^{\prime}-u_{\varepsilon}^{\prime}\right) \\
\quad+\left[f_{\xi}\left(x, u, u_{\varepsilon}^{\prime}\right)-f_{\xi}\left(x, u_{m}, u_{\varepsilon}^{\prime}\right)\right] \cdot u_{\varepsilon}^{\prime} .
\end{aligned}
$$

By (25) and (26), we have

$$
\begin{aligned}
\int_{\Omega^{\prime}} & {\left[f\left(x, u_{m}, u_{m}^{\prime}\right)-f\left(x, u, u^{\prime}\right)\right] d x } \\
\geq & \int_{\Omega^{\prime}}\left[f_{\xi}\left(x, u_{m}, u_{\varepsilon}^{\prime}\right) \cdot u_{m}^{\prime}-f_{\xi}\left(x, u, u_{\xi}^{\prime}\right) \cdot u^{\prime}\right] d x \\
& +\int_{\Omega^{\prime}}\left[f_{\xi}\left(x, u, u_{\varepsilon}^{\prime}\right) \cdot\left(u^{\prime}-u_{\varepsilon}^{\prime}\right)\right] d x \\
& +\int_{\Omega^{\prime}}\left[f_{\xi}\left(x, u, u_{\varepsilon}^{\prime}\right)-f_{\xi}\left(x, u_{m}, u_{\varepsilon}^{\prime}\right)\right] \cdot u_{\varepsilon}^{\prime} d x \\
& +\int_{\Omega^{\prime}}\left[f\left(x, u_{m}, u_{\varepsilon}^{\prime}\right)-f\left(x, u, u_{\varepsilon}^{\prime}\right)\right] d x \\
& +\int_{\Omega^{\prime}}\left[f\left(x, u, u_{\varepsilon}^{\prime}\right)-f\left(x, u, u^{\prime}\right)\right] d x
\end{aligned}
$$


Step 3. Now, we estimate the right side of inequality (27).

By (6) and (24), we have

$$
\begin{array}{r}
\int_{\Omega^{\prime}}\left[f_{\xi}\left(x, u, u_{\varepsilon}^{\prime}\right) \cdot\left(u^{\prime}-u_{\varepsilon}^{\prime}\right)\right] d x \\
\geq-L_{1} \int_{\Omega^{\prime}}\left|u^{\prime}-u_{\varepsilon}^{\prime}\right| d x \geq-L_{1} \delta .
\end{array}
$$

Thus

$$
\lim _{\varepsilon \rightarrow 0} \int_{\Omega^{\prime}}\left[f_{\xi}\left(x, u, u_{\varepsilon}^{\prime}\right) \cdot\left(u^{\prime}-u_{\varepsilon}^{\prime}\right)\right] d x \geq 0 .
$$

Since $f(x, s, \xi)$ and $f_{\xi}(x, s, \xi)$ are continuous functions, they are bounded functions on compact subset. By Lebesgue Dominated Convergence Theorem, we obtain

$$
\begin{gathered}
\lim _{m \rightarrow+\infty} \int_{\Omega^{\prime}}\left[f_{\xi}\left(x, u, u_{\varepsilon}^{\prime}\right)-f_{\xi}\left(x, u_{m}, u_{\varepsilon}^{\prime}\right)\right] \cdot u_{\varepsilon}^{\prime} d x=0 \\
\lim _{m \rightarrow+\infty} \int_{\Omega^{\prime}}\left[f\left(x, u_{m}, u_{\varepsilon}^{\prime}\right)-f\left(x, u, u_{\varepsilon}^{\prime}\right)\right] d x=0
\end{gathered}
$$

Now, we will prove

$$
\lim _{\varepsilon \rightarrow 0} \int_{\Omega^{\prime}}\left[f\left(x, u, u_{\varepsilon}^{\prime}\right)-f\left(x, u, u^{\prime}\right)\right] d x \geq 0 .
$$

By Lemma 6, there exists a sequence of nonnegative continuous functions $f_{j}(x, s, \xi) \quad(j \in N)$, such that $f_{j}(x, s, \xi)$ is convex on $\xi$, and, for all $(x, s, \xi) \in \Omega^{\prime} \times H \times R$,

$$
\begin{gathered}
f_{j}(x, s, \xi) \leq f_{j+1}(x, s, \xi), \\
f(x, s, \xi)=\sup _{j \in \mathrm{N}} f_{j}(x, s, \xi), \\
\left|f_{j}\left(x, s, \xi_{1}\right)-f_{j}\left(x, s, \xi_{2}\right)\right| \leq M_{j}\left|\xi_{1}-\xi_{2}\right| .
\end{gathered}
$$

By Levi’s Lemma, we obtain

$$
\begin{aligned}
& \lim _{j \rightarrow+\infty} \int_{\Omega^{\prime}} f_{j}\left(x, u, u_{\varepsilon}^{\prime}\right) d x=\int_{\Omega^{\prime}} f\left(x, u, u_{\varepsilon}^{\prime}\right) d x, \\
& \lim _{j \rightarrow+\infty} \int_{\Omega^{\prime}} f_{j}\left(x, u, u^{\prime}\right) d x=\int_{\Omega^{\prime}} f\left(x, u, u^{\prime}\right) d x .
\end{aligned}
$$

In order to prove (31), we only need to prove

$$
\begin{array}{r}
\lim _{\varepsilon \rightarrow 0} \int_{\Omega^{\prime}}\left[f_{j}\left(x, u, u_{\varepsilon}^{\prime}\right)-f_{j}\left(x, u, u^{\prime}\right)\right] d x \geq 0, \\
\forall j \in N .
\end{array}
$$

By (33), we have

$$
\begin{array}{r}
\int_{\Omega^{\prime}}\left[f_{j}\left(x, u, u_{\varepsilon}^{\prime}\right)-f_{j}\left(x, u, u^{\prime}\right)\right] d x \\
\quad \geq-M_{j} \int_{\Omega^{\prime}}\left|u_{\varepsilon}^{\prime}-u^{\prime}\right| d x \geq-M_{j} \delta .
\end{array}
$$

Thus (31) holds.
Step 4. Now, we need to prove

$$
\begin{aligned}
\lim _{m \rightarrow+\infty} \int_{\Omega^{\prime}}[ & f_{\xi}\left(x, u_{m}, u_{\varepsilon}^{\prime}\right) \cdot u_{m}^{\prime} \\
& \left.-f_{\xi}\left(x, u, u_{\varepsilon}^{\prime}\right) \cdot u^{\prime}\right] d x=0 .
\end{aligned}
$$

Let

$$
\begin{gathered}
g(x, s) \triangleq f_{\xi}\left(x, s, u_{\varepsilon}^{\prime}\right), \quad x \in \Omega^{\prime}, \\
G_{m}(x) \triangleq \int_{u(x)}^{u_{m}(x)} g(x, s) d s, \quad x \in \Omega^{\prime} .
\end{gathered}
$$

By triangle inequality and (7), we have

$$
\begin{aligned}
& \left|f_{\xi}\left(y_{i}, s, u_{\varepsilon}^{\prime}\left(y_{i}\right)\right)-f_{\xi}\left(x_{i}, s, u_{\varepsilon}^{\prime}\left(x_{i}\right)\right)\right| \\
& \leq\left|f_{\xi}\left(y_{i}, s, u_{\varepsilon}^{\prime}\left(y_{i}\right)\right)-f_{\xi}\left(x_{i}, s, u_{\varepsilon}^{\prime}\left(y_{i}\right)\right)\right| \\
& \quad+\left|f_{\xi}\left(x_{i}, s, u_{\varepsilon}^{\prime}\left(y_{i}\right)\right)-f_{\xi}\left(x_{i}, s, u_{\varepsilon}^{\prime}\left(x_{i}\right)\right)\right| \\
& \leq\left|f_{\xi}\left(y_{i}, s, u_{\varepsilon}^{\prime}\left(y_{i}\right)\right)-f_{\xi}\left(x_{i}, s, u_{\varepsilon}^{\prime}\left(y_{i}\right)\right)\right| \\
& \quad+L_{1}\left|u_{\varepsilon}^{\prime}\left(y_{i}\right)-u_{\varepsilon}^{\prime}\left(x_{i}\right)\right| .
\end{aligned}
$$

By (39), condition (H2) and $u_{\varepsilon}^{\prime} \in C_{0}^{\infty}(\Omega)$, we know that $g(x, s)$ is a locally absolute continuous function about $x$. So $g(x, s)$ is almost everywhere differentiable; that is, $\partial g / \partial x$ exists almost everywhere. Taking derivatives in both sides of (38), we obtain

$$
\begin{aligned}
G_{m}^{\prime}(x)= & g\left(x, u_{m}\right) \cdot u_{m}^{\prime}-g(x, u) \cdot u^{\prime} \\
& +\int_{u(x)}^{u_{m}(x)} \frac{\partial g}{\partial x} d s, \quad \text { a.e. } x \in \Omega^{\prime} .
\end{aligned}
$$

Because $G_{m}(x)$ vanishes outside $\Omega^{\prime}$, we obtain

$$
\int_{\Omega^{\prime}} G_{m}^{\prime}(x) d x=0
$$

By (40), we have

$$
\begin{array}{r}
\left|\int_{\Omega^{\prime}}\left[f_{\xi}\left(x, u_{m}, u_{\varepsilon}^{\prime}\right) \cdot u_{m}^{\prime}-f_{\xi}\left(x, u, u_{\varepsilon}^{\prime}\right) \cdot u^{\prime}\right] d x\right| \\
\quad=\left|\int_{\Omega^{\prime}}\left[g\left(x, u_{m}\right) \cdot u_{m}^{\prime}-g(x, u) \cdot u^{\prime}\right] d x\right| \\
\quad=\left|-\int_{\Omega^{\prime}} \int_{u(x)}^{u_{m}(x)} \frac{\partial g}{\partial x} d s d x\right| \leq \int_{D_{m}}\left|\frac{\partial g}{\partial x}\right| d x d s,
\end{array}
$$

where

$$
\begin{aligned}
D_{m} & =\left\{(x, s) \in \Omega^{\prime} \times H \mid \min \left\{u_{m}(x), u(x)\right\}\right. \\
& \left.\leq s(x) \leq \max \left\{u_{m}(x), u(x)\right\}\right\} .
\end{aligned}
$$

We note

$$
\begin{aligned}
\left|D_{m}\right| & =\left|\int_{\Omega^{\prime}} \int_{u}^{u_{m}} d s d x\right| \\
& \leq \int_{\Omega^{\prime}}\left|u_{m}-u\right| d x \longrightarrow 0 \quad(m \longrightarrow+\infty) .
\end{aligned}
$$


By Fubini's Theorem, we have

$$
\int_{\Omega \times R}\left|\frac{\partial g}{\partial x}\right| d x d s=\int_{H} d s \int_{\Omega \prime}\left|\frac{\partial g}{\partial x}\right| d x .
$$

Since $g(x, s)$ is absolutely continuous about $x, \partial g / \partial x$ is integrable and absolutely integrable with respect to $x$; that is,

$$
\int_{\Omega^{\prime}}\left|\frac{\partial g}{\partial x}\right| d x<+\infty
$$

By (17) and (46), we obtain

$$
\int_{\Omega \times R}\left|\frac{\partial g}{\partial x}\right| d x d s<+\infty
$$

Because of the absolute continuity of integral, we have

$$
\lim _{m \rightarrow+\infty} \int_{D_{m}}\left|\frac{\partial g}{\partial x}\right| d x d s=0
$$

By (42), we obtain

$$
\begin{aligned}
\lim _{m \rightarrow+\infty} \mid \int_{\Omega^{\prime}} & {\left[f_{\xi}\left(x, u_{m}, u_{\varepsilon}^{\prime}\right) \cdot u_{m}^{\prime}\right.} \\
& \left.\quad-f_{\xi}\left(x, u, u_{\varepsilon}^{\prime}\right) \cdot u^{\prime}\right] d x \mid=0 .
\end{aligned}
$$

Thus we just proved (36). By (29)-(31) and (36), we have

$$
\lim _{m \rightarrow+\infty} \int_{\Omega^{\prime}}\left[f\left(x, u_{m}, u_{m}^{\prime}\right)-f\left(x, u, u^{\prime}\right)\right] d x \geq 0
$$

Thus we deduce that the functional $F(u, \Omega)$ is lower semicontinuous in $W_{\text {loc }}^{1,1}(\Omega)$ with respect to the strong convergence in $L_{\text {loc }}^{1}(\Omega)$. We complete the proof.

\section{Proof of Theorem 5}

In order to prove Theorem 5 , we will verify all the conditions in Theorem 4 under the assumptions in Theorem 5. Now we will divide three steps to complete the proof of Theorem 5.

Step 1. Similar to the first step of the proof in Theorem 4, without loss of generality, we assume that the integrand $f(x, s, \xi)$ vanishes outside a compact subset of $\Omega \times R$. Thus we assume that there exists an open set $\Omega^{\prime} \times H \subset \subset \Omega \times R$, such that

$$
f(x, s, \xi) \equiv 0, \quad \forall(x, s, \xi) \in\left(\Omega \backslash \Omega^{\prime}\right) \times(R \backslash H) \times R
$$

Let $u_{m}, u \in W_{\text {loc }}^{1,1}(\Omega)$, such that $u_{m} \rightarrow u$ in $L_{\text {loc }}^{1}(\Omega)$; we need to prove

$$
\lim _{m \rightarrow+\infty} F\left(u_{m}, \Omega^{\prime}\right) \geq F\left(u, \Omega^{\prime}\right) .
$$

By Lemma 6, there exists a function sequence $\left\{f_{j}(x, s, \xi)\right\}_{j \in N}$, such that, for all $j \in N, f_{j}$ is a continuous function on
$\Omega^{\prime} \times H \subset \subset \Omega \times R$, for all $(x, s) \in \Omega^{\prime} \times H, f_{j}(x, s, \cdot)$ is convex on $R$, and, for all $(x, s, \xi) \in \Omega^{\prime} \times H \times R$,

$$
\begin{gathered}
f_{j}(x, s, \xi) \leq f_{j+1}(x, s, \xi), \\
f(x, s, \xi)=\sup _{j \in N} f_{j}(x, s, \xi), \\
\left|f_{j}\left(x, s, \xi_{1}\right)-f_{j}\left(x, s, \xi_{2}\right)\right| \leq M_{j}\left|\xi_{1}-\xi_{2}\right|, \\
(x, s) \in \Omega^{\prime} \times H, \quad \xi_{1}, \xi_{2} \in R .
\end{gathered}
$$

Let $\eta_{\varepsilon} \in C_{c}^{\infty}(R)(0<\varepsilon \ll 1)$ be a mollifier, and define the $f_{j, \varepsilon}=f_{j} * \eta_{\varepsilon}$; that is,

$$
f_{j, \varepsilon}(x, s, \xi)=\int_{R} f_{j}(x, s, \xi-z) \eta_{\varepsilon}(z) d z .
$$

By (55), we have

$$
\begin{aligned}
& \left|f_{j, \varepsilon}(x, s, \xi)-f_{j}(x, s, \xi)\right| \\
& \quad \leq \int_{R}\left|f_{j}(x, s, \xi-z)-f_{j}(x, s, \xi)\right| \eta_{\varepsilon}(z) d z \\
& \quad \leq \int_{\operatorname{supp} \eta_{\varepsilon}} M_{j}|z| \cdot \eta_{\varepsilon}(z) d z \leq M_{j} \cdot \varepsilon .
\end{aligned}
$$

Choose $\varepsilon=\varepsilon_{j}=1 / j M_{j} \rightarrow 0$. By (57), we have

$$
\left|f_{j, \varepsilon_{j}}(x, s, \xi)-f_{j}(x, s, \xi)\right| \leq M_{j} \varepsilon_{j}=\frac{1}{j} .
$$

So

$$
\begin{aligned}
f_{j}(x, s, \xi)-\frac{2}{j} & \leq f_{j, \varepsilon_{j}}(x, s, \xi)-\frac{1}{j} \\
& \leq f_{j}(x, s, \xi) \leq f(x, s, \xi) .
\end{aligned}
$$

By (53), (54), and Levi’s Lemma, we have

$$
\begin{gathered}
\lim _{i \rightarrow+\infty} \int_{\Omega^{\prime}} f_{j}\left(x, u(x), u^{\prime}(x)\right) d x \\
=\int_{\Omega^{\prime}} f\left(x, u(x), u^{\prime}(x)\right) d x .
\end{gathered}
$$

Let

$$
F_{j}\left(u, \Omega^{\prime}\right)=\int_{\Omega^{\prime}}\left[f_{j, \varepsilon_{j}}\left(x, u(x), u^{\prime}(x)\right)-\frac{1}{j}\right] d x .
$$

By (59)-(61), we have

$$
\begin{aligned}
\lim _{j \rightarrow+\infty} F_{j}\left(u, \Omega^{\prime}\right) & =F\left(u, \Omega^{\prime}\right) \\
& =\int_{\Omega^{\prime}} f\left(x, u(x), u^{\prime}(x)\right) d x .
\end{aligned}
$$

Obviously,

$$
F_{j}\left(u, \Omega^{\prime}\right) \leq F\left(u, \Omega^{\prime}\right), \quad \forall j \in N .
$$


Thus

$$
\sup _{j \in N} F_{j}\left(u, \Omega^{\prime}\right)=F\left(u, \Omega^{\prime}\right) .
$$

Therefore $F\left(u, \Omega^{\prime}\right)$, being the supremum of the family of functionals $\left\{F_{j}\left(u, \Omega^{\prime}\right)\right\}_{j \in N}$, will be lower semicontinuous if every $\left\{F_{j}\left(u, \Omega^{\prime}\right)\right\}$ is lower semicontinuous.

Step 2. In order to prove that, for all $j \in N, F_{j}\left(u, \Omega^{\prime}\right)$ is lower semicontinuous in $W_{\text {loc }}^{1,1}(\Omega)$ with respect to the strong convergence in $L_{\text {loc }}^{1}(\Omega)$, we will prove that, for all $j \in N$, the integrand $f_{j, \varepsilon_{j}}\left(x, u(x), u^{\prime}(x)\right)$ satisfies all conditions of Theorem 4. Obviously, for all $j \in N, f_{j, \varepsilon_{j}}$ satisfies condition (H1).

For all $(x, s) \in \Omega^{\prime} \times H$ and $\xi_{1}, \xi_{2} \in R$, by (55), we have

$$
\begin{aligned}
& \left|f_{j, \varepsilon_{j}}\left(x, s, \xi_{1}\right)-f_{j, \varepsilon_{j}}\left(x, s, \xi_{2}\right)\right| \\
& \quad \leq \int_{R}\left|f_{j}\left(x, s, \xi_{1}-z\right)-f_{j}\left(x, s, \xi_{2}-z\right)\right| \cdot \eta_{\varepsilon_{j}}(z) d z \\
& \quad \leq \int_{\operatorname{supp} \eta_{\varepsilon}} M_{j}\left|\xi_{1}-\xi_{2}\right| \eta_{\varepsilon_{j}}(z) d z \leq M_{j}\left|\xi_{1}-\xi_{2}\right| .
\end{aligned}
$$

Thus

$$
\left|\frac{\partial f_{j, \varepsilon_{j}}}{\partial \xi}\right| \leq M_{j}
$$

So $f_{j, \varepsilon_{j}}$ satisfies (6) in condition (H3) of Theorem 4 .

Now, we will prove that $f_{j, \varepsilon_{j}}$ satisfies (7) in condition (H3) of Theorem 4 . By $\operatorname{supp}\left(\eta_{\varepsilon_{j}}\right) \subseteq B\left(0, \varepsilon_{j}\right)$, we have

$$
\begin{aligned}
\frac{\partial f_{j, \varepsilon_{j}}}{\partial \xi}(x, s, \xi) & =\int_{R} \frac{\partial f_{j}(x, s, \xi-z)}{\partial \xi} \cdot \eta_{\varepsilon_{j}}(z) d z \\
& =-\int_{R} \frac{\partial f_{j}(x, s, \xi-z)}{\partial z} \cdot \eta_{\varepsilon_{j}}(z) d z \\
& =\int_{R} f_{j}(x, s, \xi-z) \frac{\partial \eta_{\varepsilon_{j}}(z)}{\partial z} d z .
\end{aligned}
$$

By (55) and (67), we have

$$
\begin{aligned}
& \left|\frac{\partial f_{j, \varepsilon_{j}}}{\partial \xi}\left(x, s, \xi_{1}\right)-\frac{\partial f_{j, \varepsilon_{j}}}{\partial \xi}\left(x, s, \xi_{2}\right)\right| \\
& \quad \leq \int_{R}\left|f_{j}\left(x, s, \xi_{1}-z\right)-f_{j}\left(x, s, \xi_{2}-z\right)\right| \cdot\left|\frac{\partial \eta_{\varepsilon_{j}}(z)}{\partial z}\right| d z \\
& \quad \leq M_{j}\left|\xi_{1}-\xi_{2}\right| \cdot \int_{R}\left|\frac{\partial \eta_{\varepsilon_{j}}(z)}{\partial z}\right| d z=L_{j} M_{j}\left|\xi_{1}-\xi_{2}\right|,
\end{aligned}
$$

where

$$
L_{j}=\int_{R}\left|\frac{\partial \eta_{\varepsilon_{j}}(z)}{\partial z}\right| d z
$$

is a constant depending on $\varepsilon_{j}$. Thus $f_{j, \varepsilon_{j}}$ satisfies (7). So we proved that $f_{j, \varepsilon_{j}}$ satisfies condition $(\mathrm{H} 3)$.

Step 3. Next we will prove that $f_{j, \varepsilon_{j}}$ satisfies condition (H2).

By condition (H4), for every compact subset $\Omega^{\prime} \times H \times$ $K, f(x, s, \xi)$ is absolutely continuous about $x$, that is, for all $\varepsilon_{0}>0, \exists \delta>0$ such that for any finite disjoint open interval $\left\{\left(x_{i}, y_{j}\right)\right\}_{i=1}^{n}$ in $\Omega^{\prime}$, when $\sum_{i=1}^{n}\left(y_{i}-x_{i}\right)<\delta$, we have

$$
\sum_{i=1}^{n}\left|f\left(y_{i}, s, \xi\right)-f\left(x_{i}, s, \xi\right)\right|<\varepsilon_{0} .
$$

By Step 1, $\left\{f_{j}(x, s, \xi)\right\}_{j \in N}$ satisfies (53)-(55) and the following property:

$$
f_{j}(x, s, \xi)=\max _{1 \leq q \leq j}\left\{0, a_{q}(x, s)+b_{q}(x, s) \xi\right\}, \quad j \in N,
$$

where

$$
\begin{gathered}
a_{q}(x, s)=\int_{R} f(x, s, \rho)\left[2 \eta_{q}(\rho)+\rho \frac{\partial \eta_{q}(\rho)}{\partial \rho}\right] d \rho, \\
b_{q}(x, s)=-\int_{R} f(x, s, \rho) \frac{\partial \eta_{q}(\rho)}{\partial \rho} d \rho,
\end{gathered}
$$

And, for all $(x, s, \xi) \in \Omega^{\prime} \times H \times R, \eta_{q} \in C_{c}^{\infty}(R)(q \in N)$ are mollifiers satisfying $\eta_{q} \geq 0, \int_{R} \eta_{q}(\rho) d \rho=1$, and $\operatorname{supp}\left(\eta_{q}\right) \subseteq$ $B(0,1)$, for all $j \in N$. By (71), without of loss generality, we assume that there exists $l \in\{1, \ldots, j\}$, such that

$$
f_{j}(x, s, \xi)=a_{l}(x, s)+b_{l}(x, s) \cdot \xi,
$$

where $a_{l}, b_{l}$ are given by (72). By (70), we obtain

$$
\begin{aligned}
\sum_{i=1}^{n}\left|a_{l}\left(y_{i}, s\right)-a_{l}\left(x_{i}, s\right)\right| & \\
\leq & \int_{R} \sum_{i=1}^{n}\left|f\left(y_{i}, s, \rho\right)-f\left(x_{i}, s, \rho\right)\right| \\
& \cdot\left[2 \eta_{l}(\rho)+\left|\rho \frac{\partial \eta_{l}(\rho)}{\partial \rho}\right|\right] d \rho \\
\leq & \varepsilon_{0} \int_{B(0,1)}\left[2 \eta_{l}(\rho)+\left|\rho \frac{\partial \eta_{l}(\rho)}{\partial \rho}\right|\right] d \rho \\
\leq & \left(2+A_{l}\right) \cdot \varepsilon_{0},
\end{aligned}
$$

where

$$
A_{l}=\int_{B(0,1)}\left|\frac{\partial \eta_{l}(\rho)}{\partial \rho}\right| d \rho
$$


is a constant. Similar to the above proof, we have

$$
\begin{aligned}
& \sum_{i=1}^{n}\left|b_{l}\left(y_{i}, s\right)-b_{l}\left(x_{i}, s\right)\right| \\
& \quad \leq \int_{R} \sum_{i=1}^{n}\left|f\left(y_{i}, s, \rho\right)-f\left(x_{i}, s, \rho\right)\right| \cdot\left|\frac{\partial \eta_{l}(\rho)}{\partial \rho}\right| d \rho \\
& \quad \leq \varepsilon_{0} \int_{B(0,1)}\left|\frac{\partial \eta_{l}(\rho)}{\partial \rho}\right| d \rho \leq A_{l} \cdot \varepsilon_{0} .
\end{aligned}
$$

Thus

$$
\begin{aligned}
\sum_{i=1}^{n} \mid & f_{j}\left(y_{i}, s, \xi\right)-f_{j}\left(x_{i}, s, \xi\right) \mid \\
\leq & \sum_{i=1}^{n}\left|a_{l}\left(y_{i}, s\right)-a_{l}\left(x_{i}, s\right)\right| \\
& +\sum_{i=1}^{n}\left|b_{l}\left(y_{i}, s\right)-b_{l}\left(x_{i}, s\right)\right| \cdot|\xi| \\
\leq & \left(2+A_{l}\right) \varepsilon_{0}+A_{l} \varepsilon_{0} K_{1}=\left(2+A_{l}+A_{l} K_{1}\right) \varepsilon_{0} \triangleq \sigma .
\end{aligned}
$$

Since $\xi$ belongs to a compact set, then $K_{1}=\sup _{\xi}\{|\xi|\}<+\infty$. Choose $\varepsilon_{0}$ sufficient small, so that $\sigma$ is small enough. Thus $f_{j}(x, s, \xi)$ is absolutely continuous about $x$ for all $(x, s, \xi) \in A$, which is a compact subset of $\Omega \times R \times R$. By (56) and (77), we have

$$
\begin{aligned}
& \sum_{i=1}^{n}\left|f_{j, \varepsilon_{j}}\left(y_{i}, s, \xi\right)-f_{j, \varepsilon_{j}}\left(x_{i}, s, \xi\right)\right| \\
& \quad \leq \int_{R} \sum_{i=1}^{n}\left|f_{j}\left(y_{i}, s, \xi-z\right)-f_{j}\left(x_{i}, s, \xi-z\right)\right| \cdot \eta_{\varepsilon_{j}}(z) d z \\
& \quad \leq \sigma \cdot \int_{B\left(0, \varepsilon_{j}\right)} \eta_{\varepsilon_{j}}(z) d z=\sigma .
\end{aligned}
$$

By (67) and (78), we obtain

$$
\begin{aligned}
& \sum_{i=1}^{n}\left|\frac{\partial f_{j, \varepsilon_{j}}}{\partial \xi}\left(y_{i}, s, \xi\right)-\frac{\partial f_{j, \varepsilon_{j}}}{\partial \xi}\left(x_{i}, s, \xi\right)\right| \\
& \quad \leq \int_{R} \sum_{i=1}^{n}\left|f_{j}\left(y_{i}, s, \xi-z\right)-f_{j}\left(x_{i}, s, \xi-z\right)\right| \cdot\left|\frac{\partial \eta_{\varepsilon_{j}}(z)}{\partial z}\right| d z \\
& \quad \leq \sigma \int_{R}\left|\frac{\partial \eta_{\varepsilon_{j}(z)}}{\partial z}\right| d z=L_{j} \sigma,
\end{aligned}
$$

where $L_{j}$ are constants depending on $\varepsilon_{j}$ and given by (69) (for all $j \in N$ ). By (79), for every compact subset on $\Omega \times$ $R \times R, \partial f_{j, \varepsilon_{j}} / \partial \xi$ is absolutely continuous about $x$. Thus $f_{j, \varepsilon_{j}}$ satisfies condition $(\mathrm{H} 2)$.

Now, we have proved $f_{j, \varepsilon_{j}}$ satisfies all conditions in Theorem 4 , so $F_{j}\left(u, \Omega^{\prime}\right)$ is lower semicontinuous in $W_{\text {loc }}^{1,1}(\Omega)$ with respect to the strong convergence in $L_{\text {loc }}^{1}(\Omega)$. Thus $F(u, \Omega)$ has the same lower semicontinuity. This completes the proof of Theorem 5 .

\section{Acknowledgments}

The authors would like to thank editors for their hard work and the anonymous referees for their valuable comments and suggestions. This article is supported partially by NSF of China (11071175) and the Ph.D. Programs Foundation of Ministry of Education of China.

\section{References}

[1] R. A. Adams and J. F. Fournier, Sobolev Space, Academic Press, New York, NY, USA, 2nd edition, 2003.

[2] C. Y. Pauc, La Méthode Métrique en Calcul des Variations, Hermann, Paris, France, 1941.

[3] J. Serrin, "On the definition and properties of certain variational integrals," Transactions of the American Mathematical Society, vol. 101, pp. 139-167, 1961.

[4] L. Ambrosio, N. Fusco, and D. Pallara, Functions of Bounded Variation and Free Discontinuity Problems, Oxford Mathematical Monographs, Oxford University Press, New York, NY, USA, 2000.

[5] V. de Cicco and G. Leoni, "A chain rule in $L^{1}(\operatorname{div} ; \Omega)$ and its applications to lower semicontinuity," Calculus of Variations and Partial Differential Equations, vol. 19, no. 1, pp. 23-51, 2004.

[6] I. Fonseca and G. Leoni, "Some remarks on lower semicontinuity," Indiana University Mathematics Journal, vol. 49, no. 2, pp. $617-635,2000$.

[7] I. Fonseca and G. Leoni, "On lower semicontinuity and relaxation," Proceedings of the Royal Society of Edinburgh A, vol. 131, no. 3, pp. 519-565, 2001.

[8] M. Gori and P. Marcellini, "An extension of the Serrin's lower semicontinuity theorem," Journal of Convex Analysis, vol. 9, no. 2, pp. 475-502, 2002.

[9] M. Gori, F. Maggi, and P. Marcellini, "On some sharp conditions for lower semicontinuity in L1," Differential and Integral Equations, vol. 16, no. 1, pp. 51-76, 2003.

[10] E. de Giorgi, Teoremi di semicontinuit'a nel calcolo delle variazioni, Istituto Nazionale di Alta Matematica, Rome, Italy, 1968. 


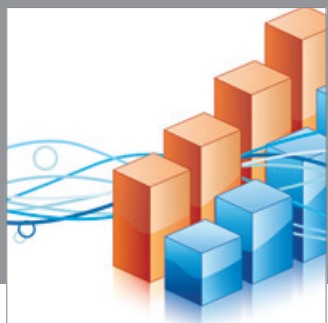

Advances in

Operations Research

mansans

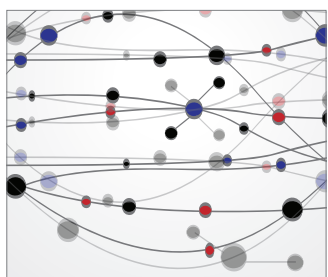

The Scientific World Journal
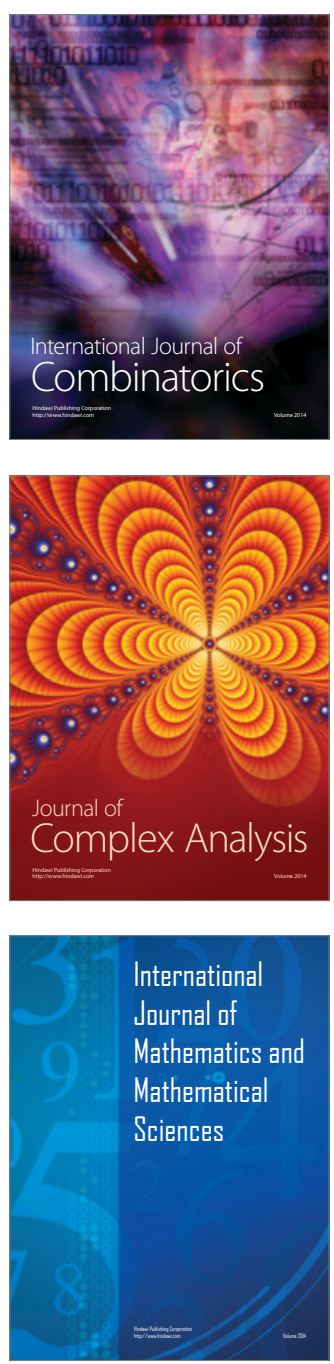
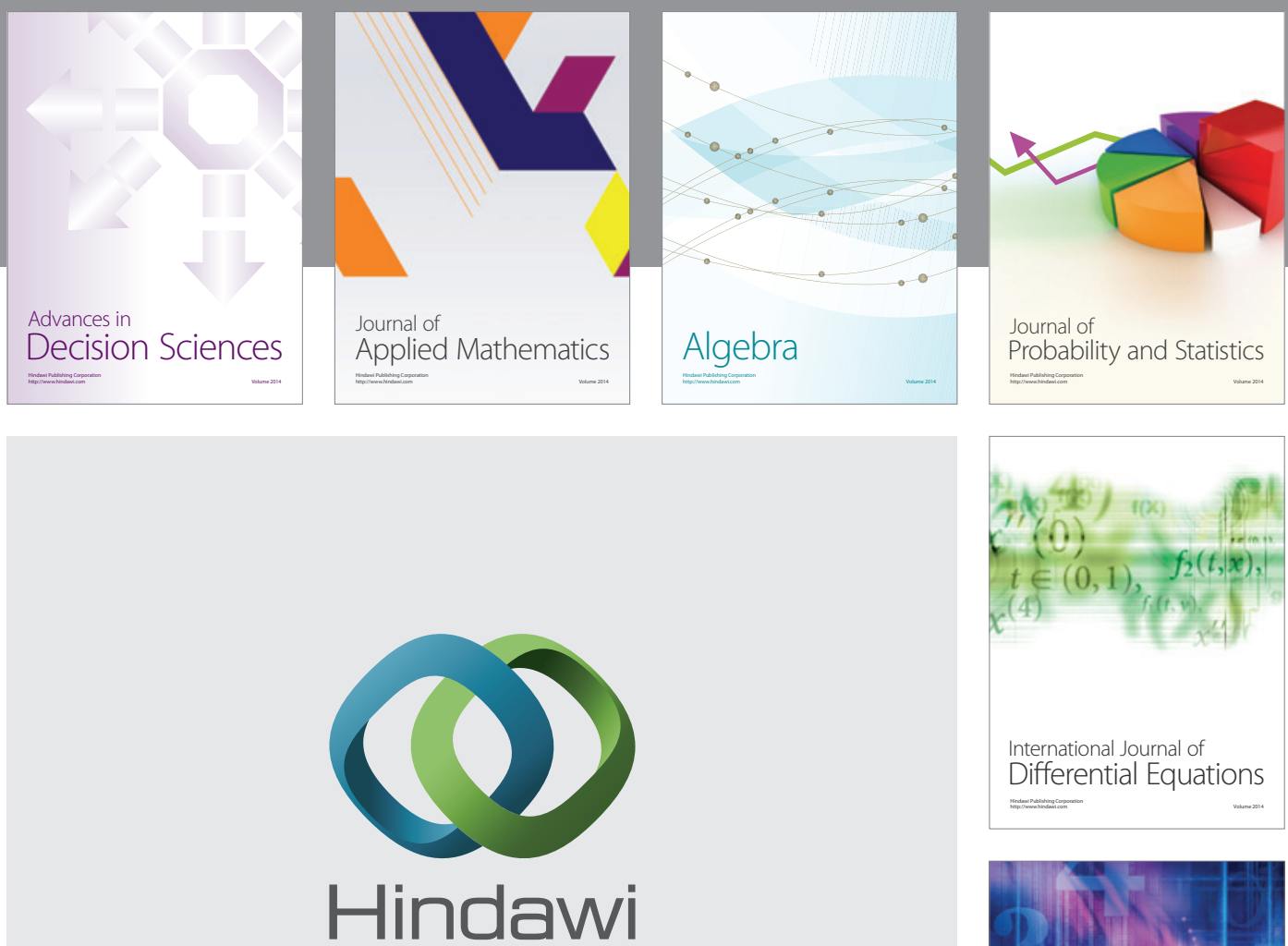

Submit your manuscripts at http://www.hindawi.com
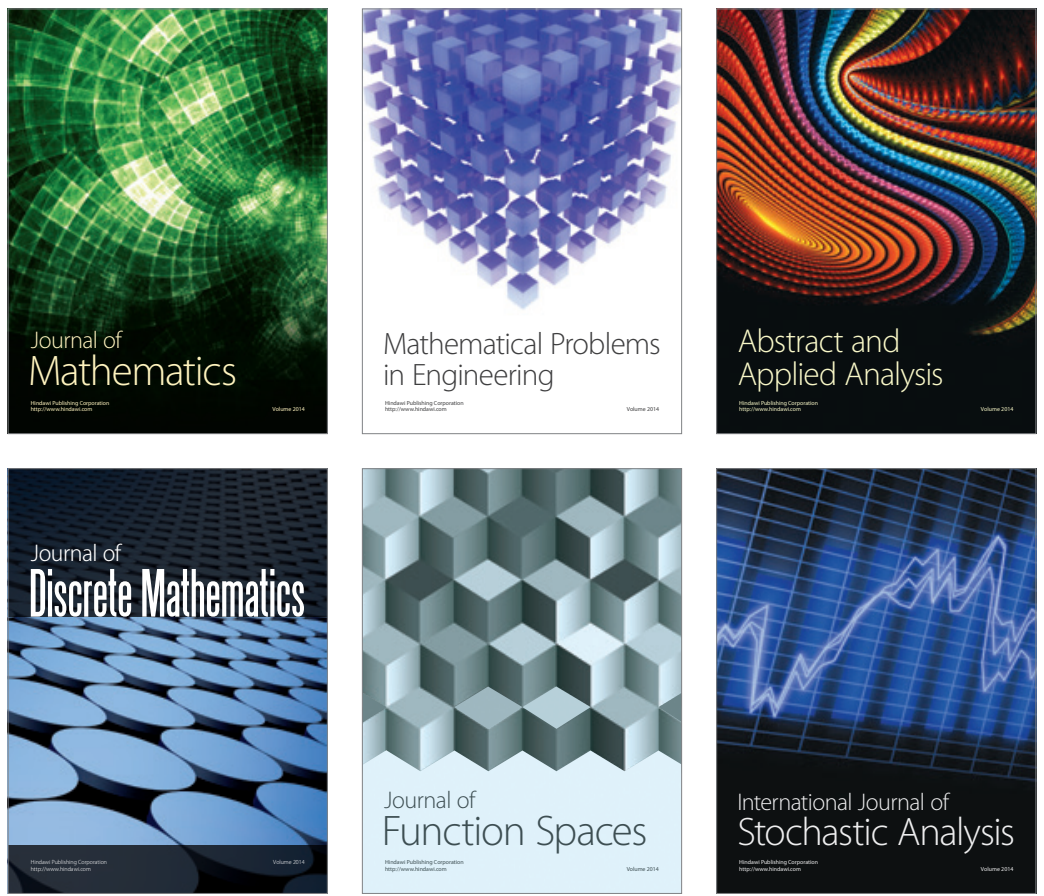

Journal of

Function Spaces

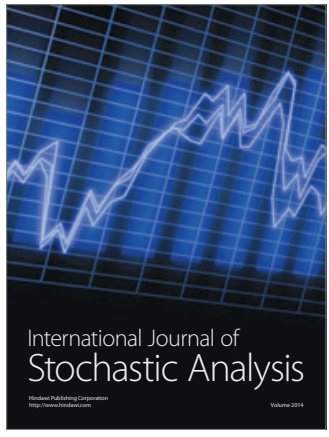

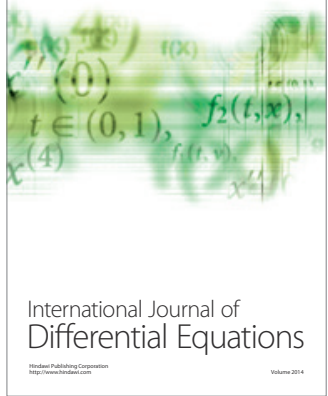
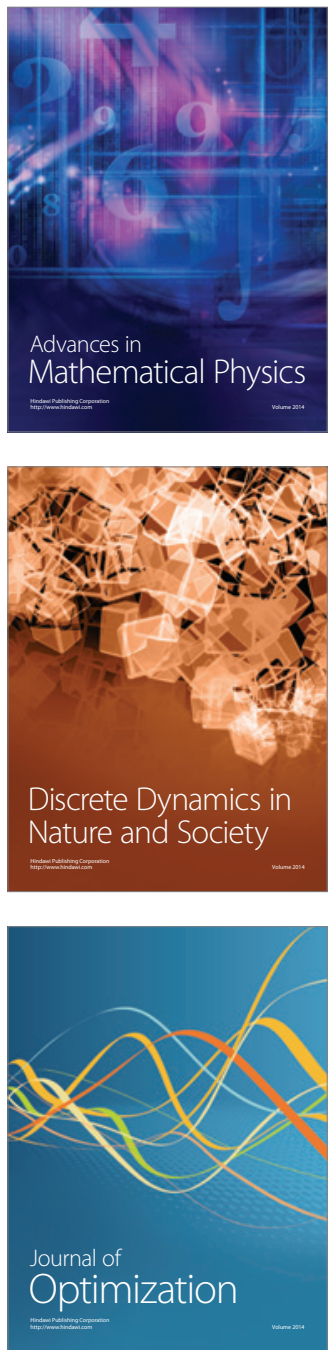